\title{
Artificial intelligence based handover decision and network selection in heterogeneous internet of vehicles
}

\author{
Shaik Mazhar Hussain ${ }^{1}$, Kamaludin Mohamad Yusof ${ }^{2}$, Rolito Asuncion ${ }^{3}$, Shaik Ashfaq Hussain ${ }^{4}$ \\ ${ }^{1,2,4}$ Department of Communications \& Advanced Telecommunication Technology, Universiti Teknologi Malaysia \\ (UTM), Malaysia \\ ${ }^{1,2,4}$ School of Electrical Engineering, Faculty of Engineering, Universiti Teknologi Malaysia, Malaysia \\ ${ }^{1,3}$ Middle East College, Muscat, Oman
}

\begin{tabular}{l}
\hline Article Info \\
\hline Article history: \\
Received Jan 2, 2021 \\
Revised Apr 12, 2021 \\
Accepted Apr 20, 2021
\end{tabular}

Keywords:

4G LTE

$5 \mathrm{G}$

DSRC

Internet of vehicles

mmWave

Network selection

Vertical handover

\begin{abstract}
Internet of vehicles (IoV) is an emerging area that gives support for vehicles via internet-assisted communication. IoV with $5 \mathrm{G}$ provides ubiquitous connectivity due to the participation of more than one radio access network. The mobility of vehicles demands to make handover in such heterogeneous networks. Usually, the previous works will directly select the network for handover or it connects with available radio access. Due to this, the occurrence of handover takes place frequently. In this paper, the integration of DSRC, LTE, and 5G mmwave on IoV is incorporated with novel handover decision making, network selection and routing algorithms. The handover decision is to ensure whether there is a need for vertical handover by using a dynamic Q-learning algorithm that uses an entropy function for threshold prediction as per the current characteristics of the environment. Then the network selection is based on fuzzy-convolution neural network that creates fuzzy rules from signal strength, distance, vehicle density, data type and line of sight. $\mathrm{V} 2 \mathrm{~V}$ chain routing is proposed to select $\mathrm{V} 2 \mathrm{~V}$ pairs using the jellyfish optimization algorithm that takes into account of the channel, vehicle characteristics, and transmission metrics. The proposed algorithms are then validated and compared with the existing state-of-the-art using OMNeT++ that combines with SUMO which gives real-time mapbased architecture. The results indicate the superiority of the proposed algorithms in comparison with the existing state-of-the-art.
\end{abstract}

This is an open access article under the CC BY-SA license.

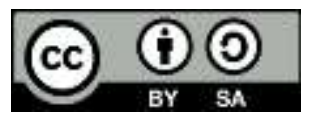

\section{Corresponding Author:}

Shaik Mazhar Hussain

Department of Communications \& Advanced Telecommunication Technology

Universiti Teknologi Malaysia

Malaysia, Johor Bahru, Skudai-81300

Email: hussain@graduate.utm.my

\section{INTRODUCTION}

Internet of vehicles (IoV) is an emerging vehicle communication via internet access. IoV is developed by integrating the intelligent transportation system (ITS) and the internet of things (IoT). IoV integrates with heterogeneous network 5G that composes different radio access networks [1]-[4]. In general, IoV vehicles employs IEEE $802.11 \mathrm{p}$ for processing which is reliable for periodic data transmission but still it is insufficient to serve spectrum usages. Also, the DSRC is not capable for long distance communication and large size data transmission. Due to this reason IoV joins 5G Millimeter-wave (mmWave) that provides high data rate for transmissions. The common issue with mmWave is blockage, because of its inability to penetrate through objects [5], [6]. The integration of IoV-5G gives ultra-high speed transmission and 
ubiquitous connectivity for vehicles. On the other hand, the LTE is able to support long distance communication. Therefore, each access network has a set of benefits as well as limitations. The combination of radio access network (RAN) in vehicles with multiple terminals which enables to switch between RAN. So that, the process of switching from one network to different type of network is termed as vertical handover (VHO) [7]-[9]. 5G network consists of macrocell, femtocell and microcell, which has different RAN in each cell. The devices in 5G supports mobility management, so they get connected with RAN in the cell. In some cases, each cell will have more than one RAN, hence it requires selection of a best network. The network selection takes place by using multi criteria decision making algorithms and others [10]-[13]. In IoV, the vehicles move at high speed and hence there is frequent change in connectivity as well as topology. Due to the change in topology, the data transmission using DSRC depends on routing [14]. The routes are selected using Q-learning, sweep algorithm and deep reinforcement learning algorithm [15]-[18]. In [14] the authors have proposed a cluster based handoff and proposed dynamic edge-backup node (DEBCK). The drawback in this work is the failure of backup mobile edge-node or cluster head will lead to poor handoff or it may not allow to perform handoff whenever there is a need. A multi-tier heterogeneous network with the focus of network selection is presented [15]. In this work, the vehicles were shortlisted from the estimated direction and then the residence time was estimated. Further from the ranking list, the network was selected. According to this work, all the handover requested vehicles will perform network selection even if the current connectivity is stronger. In [16], the authors have proposed multi-criteria utility-based network selection approach. The available network list is gathered and the weight value was computed from the metrics and then compute energy efficiency for each network. According to the score value and the user demand, the network was selected. For each request this sequential process was performed one after the other.

A two-sided one-to-many matching algorithm was proposed by authors in [16]. Analytical hierarchy process (AHP) was used to compute the weight as per the service type. This work results to increase the number of handover when the vehicle density increases. An optimal game approach was presented in [17], for network selection using probabilistic strategy. The main limitation of using game theory is that it operates with assumptions and also the initial threshold was fixed static.

In [19], ant colony optimization (ACO) algorithm is used with the idea of coloring vehicles. This work failed to consider significant parameters of vehicles in computation of the pheromone value which decides the route for transmission. In [20], the authors have proposed a cluster based adept cooperative algorithm (CACA) that mainly focuses on the QoS metrics. As per this work the process of clustering takes place and a cluster head was selected. This work follows to use optimized link state routing (OLSR) protocol with the multi point relay (MPR). However, MPR is taken in account, the vehicle will use only one as its relay for transmission. A multi-criteria decision making algorithm of AHP was developed to give preference for the vehicle traffic and the coalitional game (CG) that was used to identify the relationship between the relay vehicles, further relay selection takes place by the heuristic [21]. The main aim of this work was to solve ling-of-sight (LoS) problem. The used AHP algorithm subjects with the problem of rationality in providing preferences for the traffic of the vehicles. Hence it is not able to give proper scoring value for each vehicle that leads to poor selection of relay. A delay-aware grid-based geographic routing (DGGR) was presented in [22] that performs road weight evaluation (RWE) scheme and division of grid zones (GZ). The RWE scheme considers two main information as traffic and link. The delay was the only metric taken in account for selecting a route which is not sufficient. Routing was also performed based on optimization algorithm. In [23] a hybrid optimization that combines monarch butterfly and grey wolf optimization for route selection. The traditional issue in grey wolf optimization was not able to perform well and hence the accuracy was low. Fuzzy logic was also used for selection routes by estimating link quality and achievable throughput [23]. This work fails to tolerate mobility issues in vehicular communication. On behalf of the major limitations in the previous works, the proposed work overcomes the critical issues in routing as well as in handover and network selection.

\section{THE PROBLEM}

In this section, the problem in handover, network selection and routing is illustrated from prior research work. In [24], the author uses DSRC for V2V and mmWave for V2I communication. For DSRC communication its uses reinforcement learning algorithm i.e. Q-learning which transfer high priority and time aware V2V communication. Then TOPSIS, k-nearest neighbor (K-NN) and AHP was used for handoff decision using bandwidth, network cost, preferences, connectivity probability and signal to noise ratio (SNR) [25], [26]. TOPSIS algorithm follows the distance principle which leads to abnormality in ranking results. Also this algorithm is subjected to rank reversal problem which either includes or eliminates the order of preferences. On behalf of this problem, it performs poor to make vertical handover decisions. As per the 
ranking result, the handover is performed by the vehicle. But the need for handover is not evaluated. In contrast, if all the vehicles require handover, then the TOPSIS has to be performed individual for each vehicle since the parameters differs for each vehicle. The process of routing was performed using Dijkstra algorithm and random relay selection for data forwarding [26], [27]. Quality of service (QoS) parameters were estimated to select a route. The vehicles move dynamic and so the topology is managed by the graphs construction. The major problem stated in routing is illustrated as,

a) The parameters for graph is completely based on the past transmission of the vehicle, while the transmission of the vehicle depends on the channel metrics. The graph using these metrics is not able to predict the strength of a vehicle with its neighboring vehicle. Due to this, it causes frequent handover.

b) The maintenance of graph in heterogeneous network is complex, since the devices are not same at mobility, hence it requires larger resource and dynamic processing to manage the graph.

c) The random selection of access network with individual parameter leads to poor selection of network. Since the main constrains of QoS in this work is bandwidth or delay, i.e. it considers any one from this and hence the network selection is poor. Therefore, it minimizes QoS in the network.

The above defined problems is solved in this proposed work by novel handover decision, network selection and routing. The preference algorithm in the solutions enables to improve the performance of this proposed work.

\section{PROPOSED SOLUTION}

\subsection{Outline of the research contributions}

This section is categorized into four sub-sections to describe the environment and expand each algorithm in this proposed research work.

The main contribution of this paper is summarized as follows,

a) To mitigate the number of handover, first the decision to make handover is determined by applying Shannon entropy based Q-learning algorithm. This decision depends on the environment and the metrics are vehicle speed and signal strength.

b) The network selection using Fuzzy Convolution Neural Network in which we consider multiple metrics as distance, data type, line of sight and signal strength. In order to make this network selection process faster, we take in account of convolution neural network.

c) $\mathrm{V} 2 \mathrm{~V}$ chain routing is proposed using jellyfish optimization algorithm that computes channel metric, vehicle metrics and vehicle performance metrics. An optimal route is selected from the available routes and perform transmission.

\subsection{System model}

The proposed IoV integrated $5 \mathrm{G}$ network is designed with vehicles as shown in Figure 1. The road lane has ' $n$ ' number of moving vehicle in their own direction on the road. This environment composes of 5G base station with the use of mmWave, LTE base station, road side unit (RSU) and vehicles.
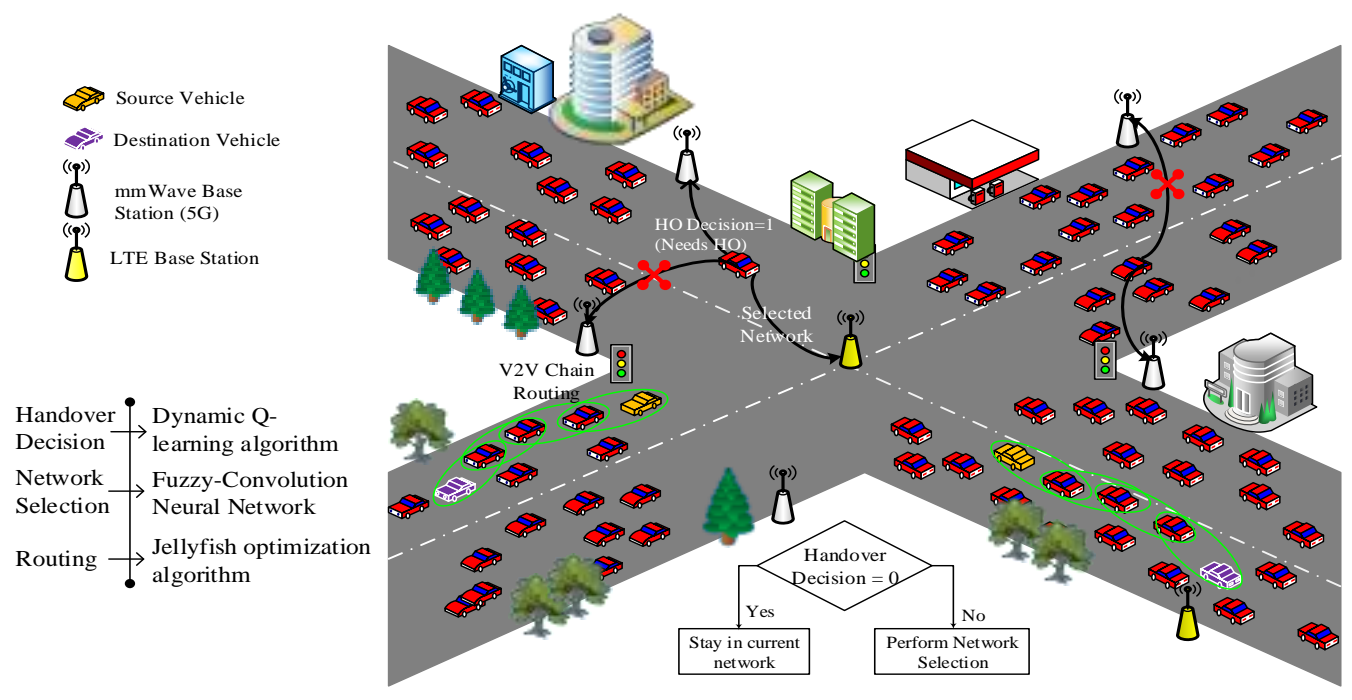

Figure 1. Proposed system model 


\subsection{Handover decision}

Handover Decision is made by Dynamic Q-learning. The two main parameters that are taken in account are vehicle speed and signal strength. Both the parameters are defined to be changing dynamic, since the vehicle speed depends on the traffic density and the signal strength differs for each RAN that are in access. However, the vehicle speed will increase and decrease within a speed limit as per the vehicle ability. Whereas the signal strength of the RAN depends on its availability and hence the signal strength value if set dynamic according to the range. For instance in range $R_{1}$ the mmWave coverage could be better than LTE, while in range $R_{2}$ the LTE will be better when compared with mmWave. Due to this, we set the threshold for signal strength in dynamic using Shannon entropy as (1).

$$
S(s s)=E[-\log (P(s s))]
$$

The Shannon entropy for signal strength is defined as $S(s s)$, that composes of $s s$ values for DSRC, mmWave and LTE that ranges between $(-30 \mathrm{dBm}$ to $-70 \mathrm{dBm})$. In equation $P(s s)$ denotes the probability of the signal strength which is present strong. The location of the vehicle will give the BSs that are in its coverage, then this threshold is set. The parameters signal strength and vehicle speed are the states that are taken in account to take an action for handover. Let $Q(S, A)$ represent state $S$ and action $A$ based on the Qvalues. Each state $S$ will have two parameters and this $Q(S, A)$ is determined and updated in the rule. The temporal difference update rule is as follows,

$$
Q(S, A)+\alpha\left(R+\gamma Q\left(S^{\prime}, A^{\prime}\right)-Q(S, A)\right) \rightarrow Q(S, A)
$$

The term $Q\left(S^{\prime}, A^{\prime}\right)$ defines next state and action $R$ is the reward given by the agent, $\gamma$ is the discount factor that is $[0-1]$, then $\alpha$ is the learning rate $[0-1]$ i.e.it denotes the step length to estimate the $(S, A)$. The action is taken using $\epsilon$-greedy policy, the $\epsilon$ represents epsilon. The pseudo code for dynamic Qlearning is here, that explains about processing of this algorithm to make decision for handover. In $\epsilon-$ greedy policy, when the probability is $(1-\epsilon)$, then the action will be taken as per the value in the Q-table. If the handover request is agreed and the action is yes, then it will select a network. Workflow of dynamic qlearning as shown in Figure 2.

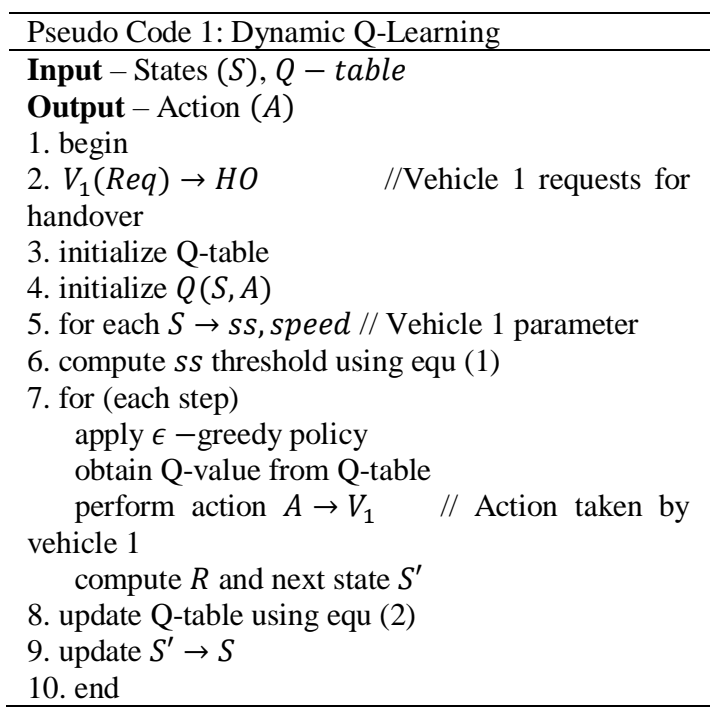

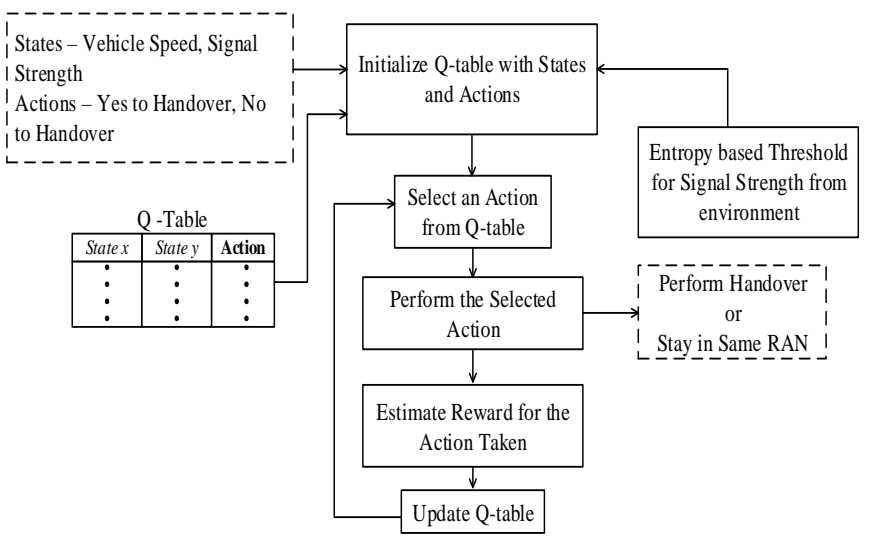

Figure 2. Workflow of dynamic q-learning

A. Network Selection: F-CNN algorithm is applied for network selection. The CNN is designed with the layers of convolution, max-pooling and fully connected layers as shown in Figure 3. The layers are employed with fuzzy rules that are defined from the metrics signal strength, distance between BS and vehicle, vehicle density in serving BS, data type (safety or non-safety) and line of sight (LoS) as shown in Table 1. Hereby, we follow this logic into CNN by considering network metrics and selects network for each vehicle. The $\mathrm{CNN}$ is able to process multiple data at a time, so a set of vehicles that needs handover is taken in account as input. 
Table 1. Fuzzy rules

\begin{tabular}{cllllll}
\multicolumn{7}{c}{ Table 1. Fuzzy rules } \\
Rule & & & Input & & Output \\
number & $S_{r}$ & Distance & $V_{D}$ & $\begin{array}{c}\text { Data } \\
\text { type }\end{array}$ & LoS & \\
\hline R1 & H & H & H & H & H & H \\
R2 & H & H & H & H & L & H \\
R3 & H & H & H & L & H & M \\
R4 & H & H & H & L & L & M \\
R5 & H & H & L & H & H & H \\
R6 & H & H & L & H & L & M \\
R7 & H & H & L & L & H & L \\
R8 & H & H & L & L & L & M \\
R9 & H & L & H & H & H & H \\
R10 & H & L & H & H & L & L \\
R11 & H & L & H & L & H & L \\
R12 & H & L & H & L & L & L \\
R13 & H & L & L & H & H & H \\
R14 & H & L & L & H & L & M \\
R15 & H & L & L & L & H & L \\
R16 & H & L & L & L & L & M \\
R17 & L & H & H & H & H & H \\
R18 & L & H & H & H & L & L \\
R19 & L & H & H & L & H & H \\
R20 & L & H & H & L & L & M \\
R21 & L & H & L & H & H & H \\
R22 & L & H & L & H & L & H \\
R23 & L & H & L & L & H & L \\
R24 & L & H & L & L & L & L \\
R25 & L & L & H & H & H & H \\
R26 & L & L & H & H & L & M \\
R27 & L & L & H & L & H & L \\
R28 & L & L & H & L & L & L \\
R29 & L & L & L & H & H & M \\
R30 & L & L & L & H & L & M \\
R31 & L & L & L & L & H & L \\
R32 & L & L & L & L & L & L \\
\hline & & & & & &
\end{tabular}

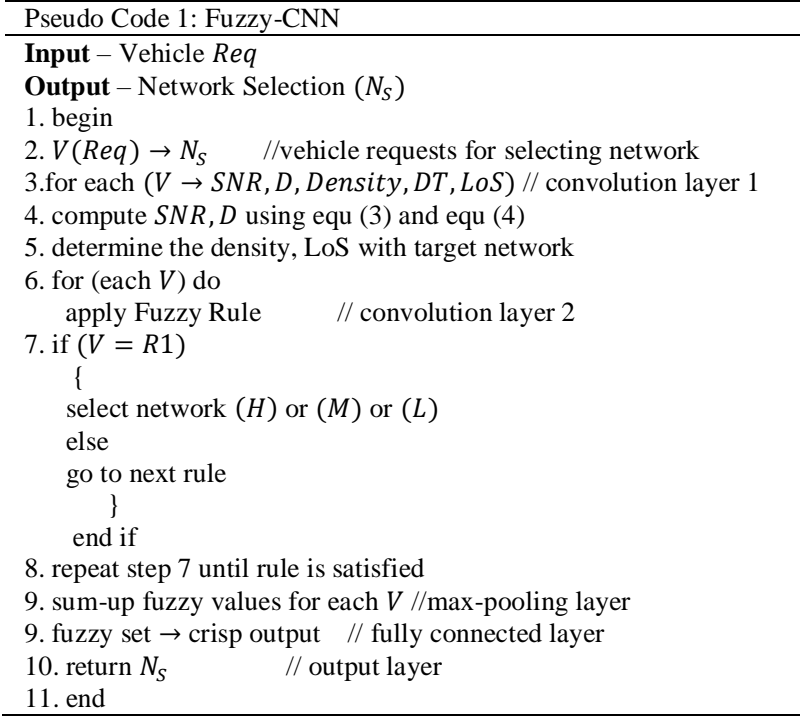

The fuzzy logic operations are built into $\mathrm{CNN}$ as shown in Figure 3. The output high $(\mathrm{H})$, medium (M) and low (L), denotes as follows,

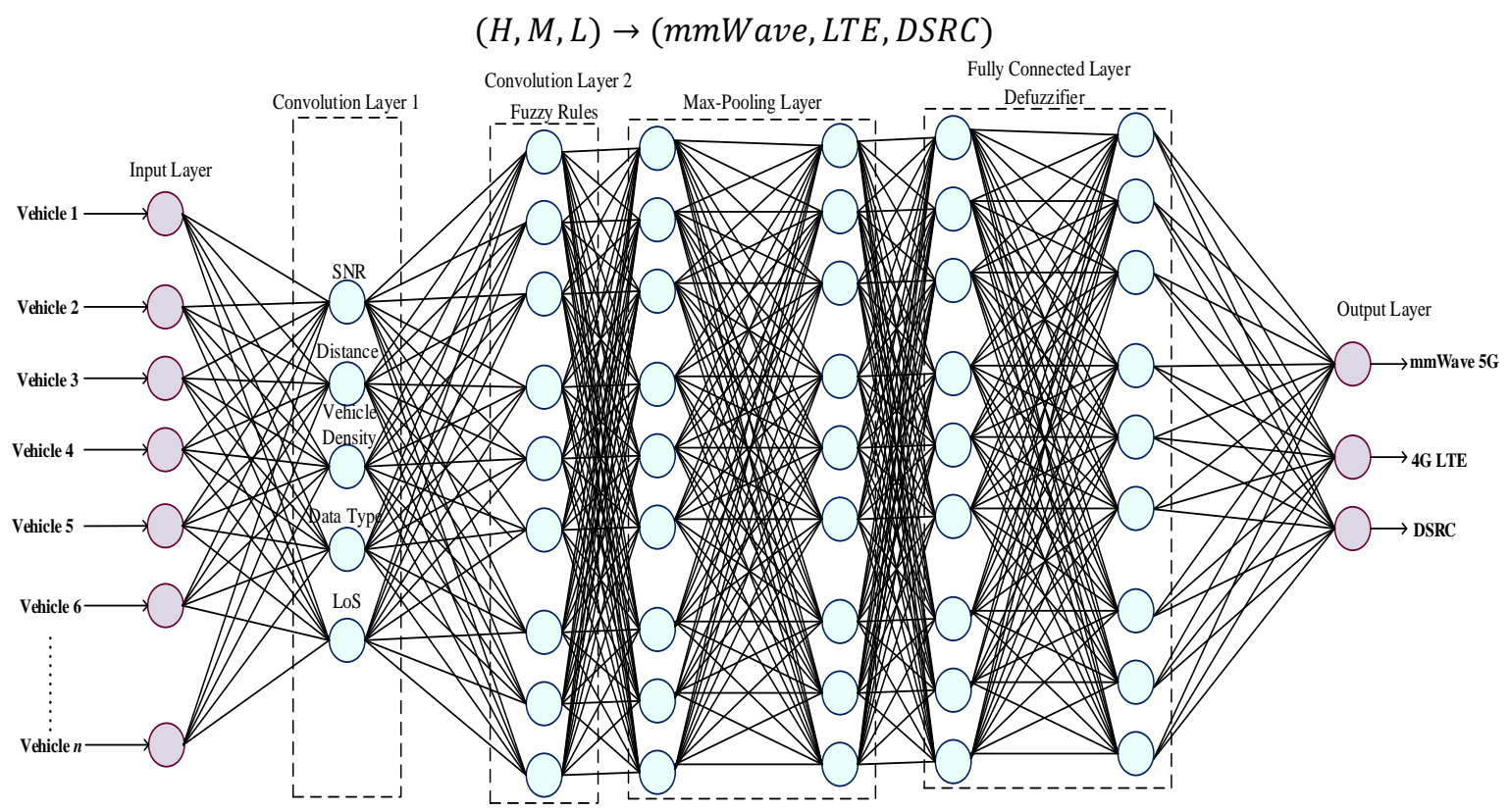

Figure 3. Fuzzy-convolutional neural network 
A pseudo code above is illustrated based on the work flow of this fuzzy-CNN algorithm.

B. Routing: V2V chain routing is the process handled perform routing by selecting V2V pairs from the source vehicle to the destination vehicle. For this communication the vehicles uses DSRC signals. The connection of $\mathrm{V} 2 \mathrm{~V}$ pairs will form a chain and so we give this as $\mathrm{V} 2 \mathrm{~V}$ chain routing. The $\mathrm{V} 2 \mathrm{~V}$ pairs are selected using jellyfish optimization algorithm. In this optimization algorithm the objective function is defined using the consideration of three sets of metrics as channel metrics ( SNR $\left(s_{r}\right)$, link quality $\left(l_{q}\right)$ ), vehicle metrics (Speed $\left(s_{p}\right)$, relative direction $\left(R_{d}\right)$ ) and vehicle performance metrics (Delay $\left(D_{l}\right)$, throughput $\left.\left(T_{p}\right)\right)$.

When a vehicle needs to forward any road traffic information, safety message it uses DSRC which is efficient to perform at short distance with low latency. Jellyfish algorithm is based on the behavior of jellyfish on ocean in search of their food. The jellyfish movement is either active or passive that is based on the ocean current or swarm. In order to switch between these two movements, a time control mechanism is used in this algorithm. The time control $c(t)$ is formulated from iteration and the random values as depicted in the following.

$$
c(t)=\left|\left(1-\frac{t}{\text { Maxite }_{i t}}\right) \times(2 * \operatorname{rand}(0,1)-1)\right|
$$

When, $\operatorname{rand}(0,1)>(1-c(t))$, then passive motion

$$
\operatorname{rand}(0,1)<(1-c(t)) \text {, then active motion }
$$

In IoV, the vehicles move faster at less traffic area i.e. in highway cases and move slower in high traffic area i.e. urban cases. Here the jellyfish is the vehicles and the ocean is the roadlane where the vehicle move in different speed.

The ocean current direction represented as $\overrightarrow{O C}$ and it is mathematically given as below,

Let,

$$
\begin{aligned}
& \overrightarrow{O C}=\frac{1}{V_{p}} \\
& =X^{*}-e_{c} \mu
\end{aligned}
$$

Then,

$$
\overrightarrow{O C}=X^{*}-d_{f f}
$$

The terms $V_{p}$ population of jellyfish in ocean, i.e. population of vehicle as per this work. $X^{*}$ denotes the best location, $\mu$ is the mean location and $e_{c}$ is the attraction factor, here the attraction of on destination. Then the objective function is defined to select a best route. This function $O F$ is formulated,

$$
O F(M s)=\sum\left(s_{r}, l_{q}\right)\left(s_{p}, R_{d}\right)\left(D_{l}, T_{p}\right)
$$

In this work a set of parameters are taken in account that is represented as Ms. The metrics delay and speed should be minimum, whereas all the other parameters can be maximum value, the vehicles that satisfy this $O F$ has higher priority to select the route. Here the $O F$ is applied for the complete route, since this work selects an optimal route from the available routes. The metrics are estimated from the channel,

$$
\begin{aligned}
& l_{q}=\frac{1}{P_{f} \times P_{r}} \\
& R_{d}=2 r \sin \sqrt{\sin ^{2}\left(\frac{\Delta_{l a}}{2}\right)+\cos \left(l a_{v}\right) * \cos \left(l a_{n p_{i}}\right) * \sin ^{2}\left(\frac{\Delta_{l n}}{2}\right)} \\
& D_{l}=\frac{P_{L}}{b}
\end{aligned}
$$

The criteria $P_{f}, P_{r}$ denotes the number of forwarded packet and the received packet in the same link between two vehicles, then $(l a, l n)$ represents the (latitude, longitude), so the vehicle location is $\left(l a_{v}, l n_{v}\right)$ 
and the next hop location is $\left(l a_{n p}, l n_{n p}\right)$ and $\mathrm{r}$ is the radius i.e. coverage of the vehicle. The estimation of delay is computed from $P_{L}, b$ that are packet length and bit rate i.e. transmission speed in bits per second. As per the defined objective function, the optimal route is selected using this jellyfish optimization algorithm. The vehicles use DSRC to perform communication over the selected route. The performance of the proposed $\mathrm{HO}$, network selection and routing is evaluated in next section.

\section{RESULT AND DISCUSSIONS}

This section is categorized into three sub-sections as simulation setup, comparative analysis and result discussion.

\subsection{Simulation setup}

This proposed work is simulated using network simulator, since vehicles are connected in network [28]. The Objective Modular Network Testbed in C++ (OMNeT++) that combines with SUMO which gives real time map based architecture. The important simulation specifications of the proposed research are shown in Table 2.

Table 2. Simulation specifications

\begin{tabular}{cc}
\hline Parameter & Range / Value \\
\hline Simulation Area & $2500 \mathrm{~m} \times 2500 \mathrm{~m}$ \\
Number of Vehicles & 100 \\
Number of 5G mmWave BSs & 2 \\
Number of 4G LTE BSs & 2 \\
Vehicle mobility type & Linear mobility \\
Vehicle Speed & 10 to $40 \mathrm{~m} / \mathrm{s}$ \\
Transmission $\quad$ DSRC & $300 \mathrm{~m}(\mathrm{Max})$ \\
Range $\quad$ LTE & $\sim 500 \mathrm{~m}$ \\
Transmission rate & $100 \mathrm{~km}(\mathrm{Max})$ \\
Packet size & - 5 packets per second \\
Simulation time & 512 bytes \\
\end{tabular}

\subsection{Comparative analysis}

This proposed work is compared with previous research work [23] in which handover network selection decision was based on TOPSIS algorithm. The parameters that are evaluated in this work are handover success probability, handover failure, unnecessary handover, throughput, delay and packet loss. Handover success probability and Handover failure is significant in validating the performance of a handover method. The increase in handover success probability denotes the better performance of the proposed algorithm. In previous work, TOPSIS was used for the selection of network that fails to perform proper ranking. The Figure 4 and 5 illustrates the success probability and failure respectively. This results shows that the decision by dynamic Q-learning and F-CNN have better selection in handover. The success probability of proposed work increases as per the increase in $\mathrm{HO}$ requests and hence this network selection is suitable for large scale environment. On the other hand, the increase in success probability leads to reduce the number of HO failure counts. According to the increase in vehicle speed, the handover failure occurs. As per the increase in the number of handover requests, the network performs to validate the need for $\mathrm{HO}$ and selection of a network. The poor performance of TOPSIS results with increase in number of unnecessary HO as shown in Figure 6.

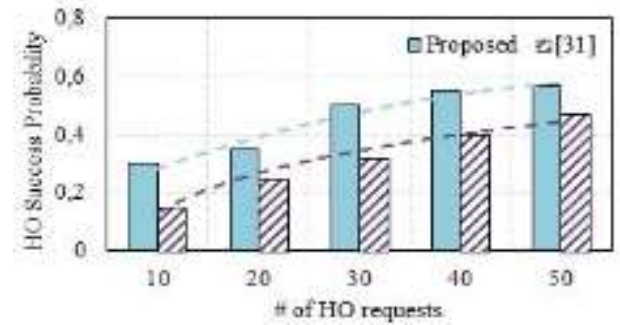

Figure 4. Comparison of $\mathrm{HO}$ success probability

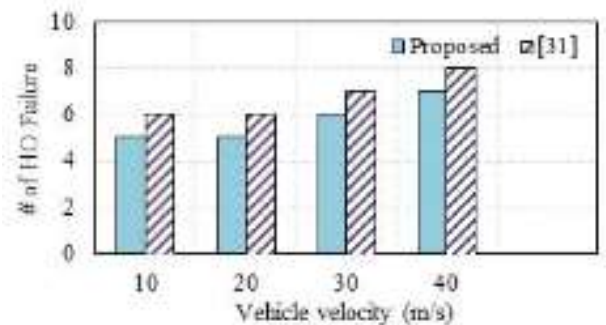

Figure 5. Comparison of $\mathrm{HO}$ failure 


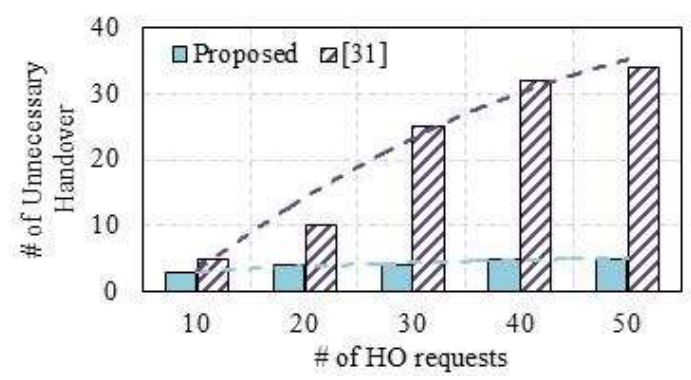

Figure 6. Comparison of unnecessary HO

Throughput and delay evaluates the network performance with respect to the capability of the defined algorithm for data transmission. The graphical plots for throughput and delay is depicted in Figure 7 and 8 that compares proposed with existing wok. On average, the throughput difference of about 20Mbps, for upto 100 number of vehicles. In proposed, increase in the result of throughput is due to the optimal selection of route using jellyfish algorithm that considers vehicle metrics, channel metrics and performance metrics all together in the objective function. The average delay difference is upto $12 \mathrm{~ms}$ for about 100 vehicles in the network. The graph shows minor growth and drop in the delay, so even the further increase in number of vehicle will not reflect on delay since the route is optimally selected.

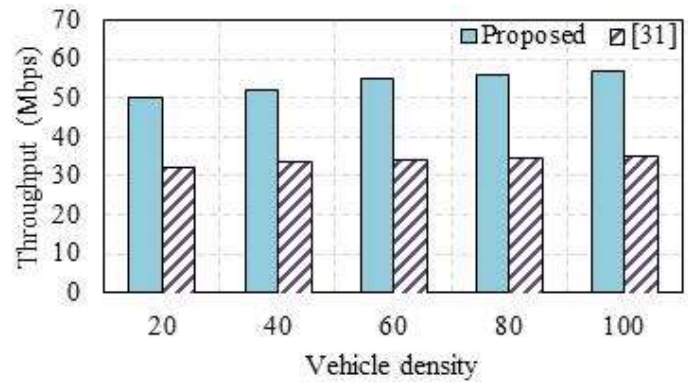

Figure 7. Comparison of throughput

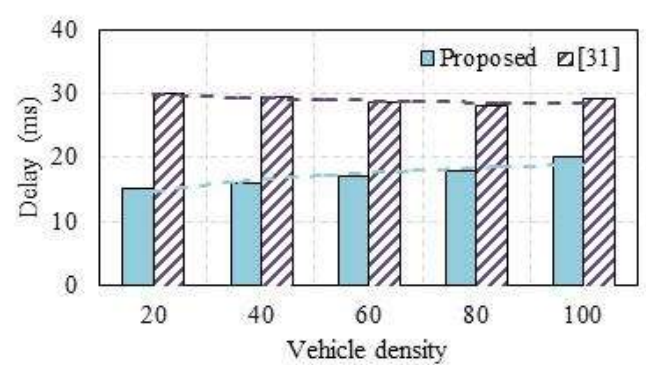

Figure 8. Comparison of delay

The smaller packet loss will intimate the better selection of route for data transmission. The packet loss in routing occur for different reasons, hence the selection of optimal route is significant. In proposed work, the route selection is by jellyfish algorithm that considers vehicle metrics, channel metrics and vehicle performance metrics which is efficient in the selection of optimal route. The graphical result of packet loss is shown in Figure 9, where the proposed work is compared and attains lesser loss than the previous routing. As per the vehicle density increases, there will higher number of transmissions, so there can be increase in packet loss. However, the vehicle density increases it also reflects on packet loss but in proposed work, the optimal selection of route leads to manage the packet loss.

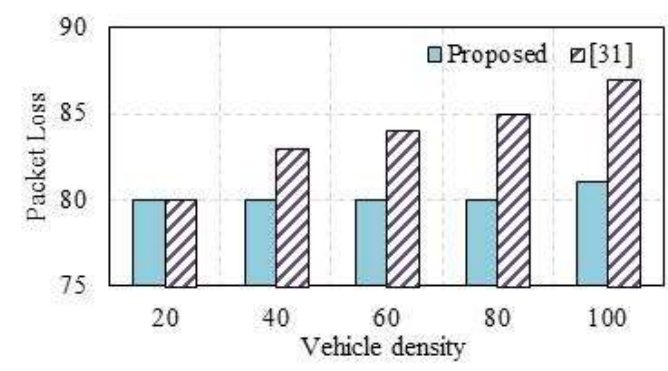

Figure 9. Comparison of packet loss 


\section{CONCLUSION}

In this paper, the IoV environment is constructed with vehicles that are equipped with three terminals as DSRC, LTE, and 5G mmwave. The data transmission requirement depends for each data type. Due to the multiple RAN, the process of handover is proposed in this work. First, a dynamic Q-learning algorithm is used for making handover decisions with the dynamic threshold computation using entropy for signal characteristics. The use of reinforcement learning algorithm for handover decision can learn the environment and make a decision that leads to minimize the number of unnecessary handovers. Then the network selection by fuzzy-CNN, since it can process multiple requests that arrive at a time. This fuzzy-CNN enables to consider multiple parameters to select the network. There are 32 fuzzy rules, based on which the network is selected. IoV is a large-scale network, to solve scalability issue in network selection, the fuzzy$\mathrm{CNN}$ is used. This algorithm can work faster due to the construction of neural nodes that are operated in parallel. When a vehicle needs to communicate with a long-distance vehicle on the road lane, a route is selected. Here DSRC is preferred by the vehicles since it is low latency when communicating with the neighboring hop vehicles. A V2V chain routing is proposed for routing that selects a route using jellyfish optimization and selects $\mathrm{V} 2 \mathrm{~V}$ pairs in a route and performs data transmission. The objective function for routing is defined from three as vehicle metrics, channel metrics, and performance metrics. In this way an optimal route is selected for data transmission. Using dynamic Q-learning algorithm, unnecessary handovers are reduced by $25 \%$ and handover failure by $2 \%$. Fuzzy CNN improves the handover success probability by appropriately selecting the network and modified jelly fish improves throughput by $15 \%-20 \%$ and minimizes delay and packet losses. Hence, the validation and the performance analysis showed the superiority of the proposed algorithm when compared to the current state-of-the-art.

\section{REFERENCES}

[1] Yang Yang, Kun Hua, "Emerging Technologies for 5G-Enabled Vehicular Networks," IEEE Access, vol. 7, pp. 181117-181141, 2019, doi: 10.1109/ACCESS.2019.2954466.

[2] Carlos Renato Storck, Fátima Duarte-Figueiredo, "A Survey of 5G Technology Evolution, Standards, and Infrastructure Associated with Vehicle-to-Everything Communications by Internet of Things," IEEE Access, vol. 8, pp. 117593-117614, 2020, doi: 10.1109/ACCESS.2020.3004779.

[3] Ramon Sanchez-Iborra, José Santa, Jorge Gallego-Madrid, Stefan Covaci, Antonio Skarmeta, "Empowering the Internet of Vehicles with Multi-RAT 5G Network Slicing," Sensors, MDPI, vol. 19, no. 14, p. 3107, 2019, doi: $10.3390 / \mathrm{s} 19143107$.

[4] Elhadja Benalia, Salim Bitam, Abdelhamid Mellouk, "Data dissemination for Internet of vehicles based on 5G communications: A survey," Transactions on Emerging Telecommunications Technologies, Future Internet of Vehicles, vol. 31, no. 5, 2020, doi: 10.1002/ett.3881

[5] Tommaso Zugno, Matteo Drago, Marco Giordani, Michele Polese, Michele Zorzi, "Toward Standardization of Millimeter-Wave Vehicle-to-Vehicle Networks: Open Challenges and Performance Evaluation," IEEE Communications Magazine, vol. 58, no. 9, pp. 79-85, 2020, doi: 10.1109/MCOM.001.2000041.

[6] Tho Minh Duong, Sungoh Kwon, "Vertical Handover Analysis for Randomly Deployed Small Cells in Heterogeneous Networks," IEEE Transactions on Wireless Communications, vol 1. 19. no. 4, pp. 2282-2292, 2020, doi: 10.1109/TWC.2019.2963829.

[7] Senthilnathan Palaniapan, Mohammed Ahsan Kollathodi, "Real time implementation of embedded devices as a security system in intelligent vehicles connected via vanets," Int J Elec \& Comp Eng, vol. 9, no. 6, pp. 4788-4797, 2019, doi: 10.11591/ijece.v9i6.pp4788-4797.

[8] Macro Giordani, Andrea Zanella, Takamasa Higuchi, Onur Altintas, Michele Zorzi, "On the Feasibility of Integrating mmWave and IEEE 802.11p for V2V Communications," IEEE 88th Vehicular Technology Conference, 2019, doi: 10.1109/VTCFall.2018.8690697.

[9] Shidrokh Goudarzi, et al., "Intelligent Technique for Seamless Vertical Handover in Vehicular Networks," Mobile Networks and Applications, vol. 23, no. 6, pp. 1462-1477, pp. 1462-1477, 2018, doi: 10.1007/s11036-017-0979-6.

[10] Ammar Bathich, Mohd Asri Mansor, Saiful Izwan Sulimn, Sinam Ghassan Abid Ali, "Q-learning vertical handover scheme in two-tier LTE-A networks," International Journal of Electrical and Computer Engineering, vol. 10, no. 6, pp. 5824-5831, 2020, doi: 10.11591/ijece.v10i6.pp5824-5831.

[11] Rishabh Mitra, Raghavendra Ganiga, "A novel approach to sensor implementation for healthcare systems using internet of things," International Journal of Electrical and Computer Engineering (IJECE), vol. 9, no. 6, pp. 50315045, 2019, doi: 10.11591/ijece.v9i6.pp5031-5045.

[12] Na Lin, Yanjun Shi, Tongliang Zhang, Xuping Wang, "An Efficient Order-Aware Hybrid Genetic Algorithm for Capacitated Vehicle Routing Problems in Internet of Things," IEEE Access, vol. 7, pp. 86102-86114, 2019, doi: 10.1109/ACCESS.2019.2925831.

[13] James J. Q. Yu, Wen Yu, Jiatao Gu, “Online Vehicle Routing With Neural Combinatorial Optimization and Deep Reinforcement Learning," IEEE Transactions on Intelligent Transportation Systems, vol. 20, no. 10, pp. 38063817, 2019, doi: 10.1109/TITS.2019.2909109. 
[14] Khalid Mahmood Awan, Malik Nadeem, Ali Safaa Sadiq, Abdullah Alghushami, Imran Khan, Khaled Rabie, "Smart Handoff Technique for Internet of Vehicles Communication using Dynamic Edge-Backup Node," Electronics, MDPI, vol. 9, no. 3, p. 524, 2020, doi: 10.3390/electronics9030524.

[15] Emmanuel Ndashimye, Nurul I. Sarkar, Sayan Kumar Ray, "A network selection method for handover in vehicleto-infrastructure communications in multi-tier networks," Wireless Networks, Springer, vol. 26, no. 1, pp. 387-401, 2020, doi: 10.1007/s11276-018-1817-x.

[16] Qi Si, Zhipeng Cheng, Yuhui Lin, Lianfen Huang, Yuliang Tang, "Network Selection in Heterogeneous Vehicular Network: A One-to-Many Matching Approach," IEEE 91 10.1109/VTC2020-Spring48590.2020.9129074.

[17] Xiangmo Zhao, Xiaochi Li, Zhigang Xu, Ting Chen, “An Optimal Game Approach for Heterogeneous Vehicular Network Selection with Varying Network Performance," IEEE Intelligent Transportation Systems Magazine, vol. 11 , no. 3, pp. 80-92, 2019.

[18] Abdulraqeb Alhammadi, et al., "An intelligent spectrum handoff scheme based on multiple attribute decision making for LTE-A network," International Journal of Electrical and Computer Engineering (IJECE), vol.9, no. 6, pp. 5330-5339, 2019, doi: 10.11591/ijece.v9i6.pp5330-5339, doi: 10.11591/ijece.v9i6.pp5330-5339.

[19] Tri-Hai Nguyen, Jason J. Jung, "ACO-based Approach on Dynamic MSMD Routing in IoV Environment," $16^{\text {th }}$ International Conference on Intelligent Environments, 2020, doi: 10.1109/IE49459.2020.9154927.

[20] Nori M. Al-Kharasani, Zuriati Ahmad Zukarnain, Shamala K. Subramaniam, Zurina Mohd Hanapi, "An Adaptive Relay Selection Scheme for Enhancing Network Stability in VANETs," IEEE Access, vol. 8, pp. 128757-128765, 2020, doi: 10.1109/ACCESS.2020.2974105

[21] Chen Chen, Lei Liu, Tie Qiu, Dapeng Oliver Wu, Zhiyuan Ren, "Delay-Aware Grid-Based Geographic Routing in Urban VANETs: A Backbone Approach,” IEEE/ACM Transactions on Networking, vol. 27, no. 6, pp. 2324-2337, 2019, doi: 10.1109/TNET.2019.2944595.

[22] G. Tony Santhosh, S. Dhandapani, "Hybridization of Monarch Butterfly and Grey Wolf Optimization for Optimal Routing in VANET," International Journal of Engineering and Advanced Technology (IJEAT), vol. 9, no. 2, 2019.

[23] Ohoud Alzamzami, Imad Mahgoub, "Fuzzy Logic-Based Geographic Routing for Urban Vehicular Networks Using Link Quality and Achievable Throughput Estimations," IEEE Transactions on Intelligent Transportation Systems, vol. 20, no. 6, pp. 2289-2300, 2019, doi: 10.1109/TITS.2018.2867177.

[24] Zhengguo Sheng, Andreas Pressas, Victor Ocheri, Falah Ali, Richard Rudd, Maziar Nekovee, "Intelligent 5G Vehicular Networks: An Integration of DSRC and mmWave Communications," International Conference on Information and Communication Technology Convergence (ICTC), IEEE, 2018, doi: 10.1109/ICTC.2018.8539687.

[25] Li Yan, et al., "Machine Learning Based Handovers for Sub-6 GHz and mmWave Integrated Vehicular Networks," IEEE Transactions on Wireless Communications, vol. 18, no. 10, pp. 4873-4885, 2019, doi: 10.1109/TWC.2019.2930193.

[26] Mohamed Lahby, AyoubEssouiri, Abderrahim Sekkaki, "A novel modeling approach for vertical handover based on dynamic k-partite graph in heterogeneous networks," Digital Communications and Networks, Sciencedirect, vol. 5, no. 4, pp. 297-307, 2019, doi: 10.1016/j.dcan.2019.10.001.

[27] Hussain, S. M., Yusof, K. M., Hussain, S. A., \& Eberechukwu N, P. A, "Review of Interoperability issues in Internet of Vehicles (IoV)," International Journal of Computing and Digital Systems, vol. 8, no. 1, pp. 73-83, 2019, doi: $10.12785 / \mathrm{ijcds} / 080108$

[28] Carlos Renato Storck, Fátima Duarte-Figueiredo, “A 5G V2X Ecosystem Providing Internet of Vehicles,” Sensors, MDPI, vol. 19, no. 3, 2019, doi: 10.3390/s19030550.

\section{BIOGRAPHIES OF AUTHORS}

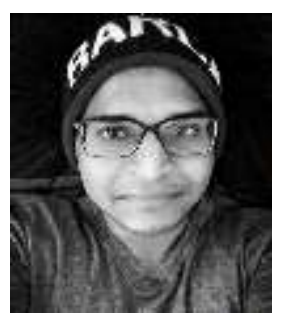

Mr. Shaik Mazhar Hussain, Department of Electronics and Communications Engineering received his Master's degree in Embedded System from India in 2012 and received PGCert from Coventry University, UK in December 2017. He is currently a Ph.D candidate at the Universiti Teknology Malaysia (UTM), Johor Bahru, Malaysia. His research areas of interest include Wireless Communications and Intelligent transportation system (ITS).

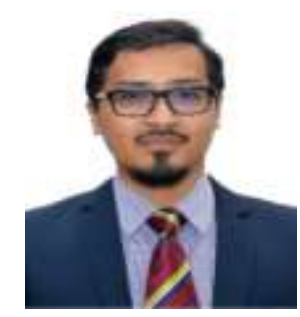

Dr. Kamaludin Mohamad Yusof is a senior lecturer at the School of Electrical Engineering, Universiti Teknologi Malaysia (UTM), Skudai Johor. Graduated with a Bachelor of Electrical Engineering (Electronics) (2000), Master of Electrical Engineering (2003) from UTM and Phd in Electrical engineering University of Essex UK (2014). He has involved in several research areas such as Internet of Thing, Internet Networking and Network Security. 


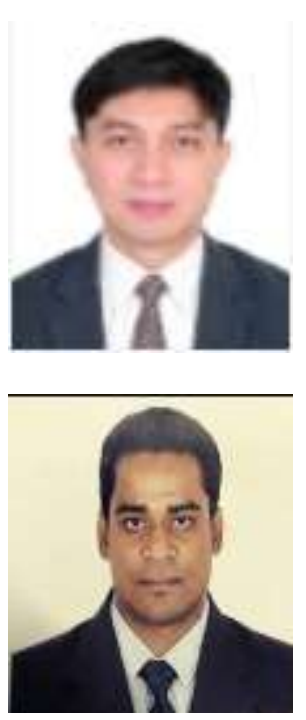

Mr. Rolito Asuncion, Department of Electronics and Communications Engineering, Middle East College, Oman. Graduated with a Bachelor of Electronics and Communication (1990) from University of Santo Tornas and Master of Electrical Engineering (2000) from Mapua Institute of Technology. His research areas of interest include Linear Control Engineering, Robotics, and PLC-SCADA.

Mr. Shaik Ashfaq Hussain, Department of Communications Engineering, Advanced Telecommunication Technology (ATT) received his Master's degree in Embedded and VLSI System design from India in 2013. He is currently a Ph.D candidate at the Universiti Teknology Malaysia (UTM), Johor Bahru, Malaysia. His Research areas of interest include Internet of Things (IoT) and Intelligent Transportation system (ITS). 